\title{
Oncopharmacogenomics of Hepatocellular Carcinoma - A Therapy Related Review
}

\author{
P K Prem Ravi Varma* \\ Head of Department, Department of Medical Oncology, Cochin Cancer Research Centre, Government Medical College Campus, India
}

Submission: December 16, 2020; Published: January 29, 2021

*Corresponding author: PK Prem Ravi Varma, Head of Department, Department of Medical Oncology, Cochin Cancer Research Centre, Government Medical College Campus, Ernakulam, Kerala State, India

\begin{abstract}
Hepatocellular carcinoma (HCC) remains a highly complex disease resistant to commonly used chemotherapy and radiotherapy. As the fifth most common cancer worldwide with the third highest mortality rate and very poorly understood molecular pathways driving hepatocarcinogenesis, new treatment strategies are urgently needed for this devastating disease. The multi kinase inhibitor Sorafenib was the first molecular targeted drug in HCC that led to significant survival benefit in patients with advanced tumors. It is the first drug to be considered standard of care for advanced HCC and supports the importance of molecular therapies in the treatment of this cancer.

Analysis of genetic and epigenetic alterations as well as different molecular pathways involved in the development of HCC help to identify potential new druggable targets. A variety of novel compounds are already under preclinical or clinical investigation, and accumulating evidence suggests that combination therapy targeting different pathways will potentiate anti-tumoral effects and will become the future therapeutic approach. In addition, the establishment of a robust molecular classification will pave the way for a more personalized treatment scheme in HCC. In this article a review of the current knowledge of the molecular pathogenesis of HCC and an overview of molecular targeted therapies in the management of HCC are provided.
\end{abstract}

\section{Introduction}

Hepatocellular carcinoma (HCC) is the fifth leading cancer in the world and more than $80 \%$ of cases occur in Asia. Even though Hepatitis B and C is the most common cause of HCC, a variety of risk factors have been identified. These include hereditary hemochromatosis, and cirrhosis of almost any cause. HCC also occurs in patients without known risk factors. Most of HCC cases develop from a cirrhotic liver, with an annual incidence of 2-6\% for hepatitis B virus carriers and 3-5\% for hepatitis C virus-infected patients. The chance of developing HCC among individuals with HBV and exposure to aflatoxin was 59 times above the normal population incidence. Survival for patients with HCC even in early stages is worse. Most (60-70\%) of the patients are diagnosed with advanced stage and having 1-2 months median survival time onwards.

HCC is the most frequent cause of all liver cancers and constitutes $90 \%$ of cancers of liver globally [1]. Even though cancers of lungs, breast and large intestine have been identified as three most common cancers, the three leading causes of annual death due to cancers include lungs, stomach and liver [1]. Approximately 7.5 Lakhs of new cases of HCC per year occurs globally which makes HCC as the 5th common cause of cancers effecting humans [1]. The mortality in HCC is very high; about 7 Lakhs death due to HCC occur annually and has been estimated to be the third common cause of death due to cancers effecting humans [1]. In India, information on HCC is inadequate. From 1988 till December 2012, over two and half decades only 68 publications have been listed in the PUBMED, all from tertiary care center, on select areas and most studies included small samples. The cancer registries in India probably do not provide accurate estimates of HCC prevalence due to its predominant urban locations and the source of information on cancers are from cytology, oncology sites, and municipal registers of death.

\section{Molecular Pathogenesis}

Complex genetic and epigenetic alterations, chromosomal aberrations, mutations, and altered molecular pathways lead to the development of HCC. The exact sequence of hepatocarcinogenesis though, including the development of pre-neoplastic lesions and its progression to HCC, is not well understood [2,3]. Cirrhosis is present in $80 \%$ of those diagnosed with HCC and is therefore 
considered as a main risk factor. Infection with HBV and HCV account for the majority of cases of HCC; however, whereas HCC in those infected with HCV is usually associated with cirrhosis, those infected with HBV can develop HCC in the absence of cirrhosis. Hepatitis $C$ virus can act as a Wnt ligand, inactivates $\mathrm{p}_{53}$ and transactivates Ras [4]. In contrast, integration of hepatitis B virus DNA into the genome induces genomic instability and genetic alterations like activation of oncogenes or silencing of Tumor Suppressor Genes (TSG) [5]. In general, any condition leading to chronic inflammation in the liver, such as chronic intake of alcohol or autoimmune hepatitis, causes genomic and mitochondrial damage to cells facilitating the development of neoplasms. In certain areas of Africa and Asia, aflatoxin B represents the most important risk factor for HCC through its strong association with $\mathrm{p}_{53}$ point mutations [6]. In addition, HCC is being increasingly recognized in individuals with chronic liver diseases associated with the Metabolic Syndrome (MS).

These abovementioned risk factors for HCC can cause initial damage to the liver, but several additional external or internal influences are needed to finally initiate the multistep process leading to the development of HCC. These events can start at the level of mature hepatocytes or liver stem cells, but in any of the circumstances, selected cell populations develop a growth advantage and proliferate into Dysplastic nodules (DN) [2]. Additional genomic hits (genetic, epigenetic alterations, mutations) are then necessary to induce a malignant phenotype in these cells. It has been proposed by Vogelstein et al. that disruption of at least three different molecular pathways is required for the development of a solid tumor [7]. Any hit can therefore be the responsible mechanism altering crucial key genes of cancer pathways, which as a whole then lead to the development of a solid malignancy. In order to fully understand the molecular pathogenesis of HCC, the following cancer pathways as well as genetic and epigenetic alterations have been studied extensively with the consequent development of molecular targeted anticancer molecules.

Signaling pathways - HCC wears many biological Hats

Wnt- $\beta$-catenin, hedgehog, c-Met, IGF, Ras-MAPK, PI3/Akt/ mTOR, and apoptosis are the most important intracellular molecular signaling pathways in HCC. Relevant signaling pathways target of therapies, and molecular targeted therapies currently tested in preclinical and clinical trials in HCC. Only sorafenib has been approved by regulatory agencies (Figure 1).

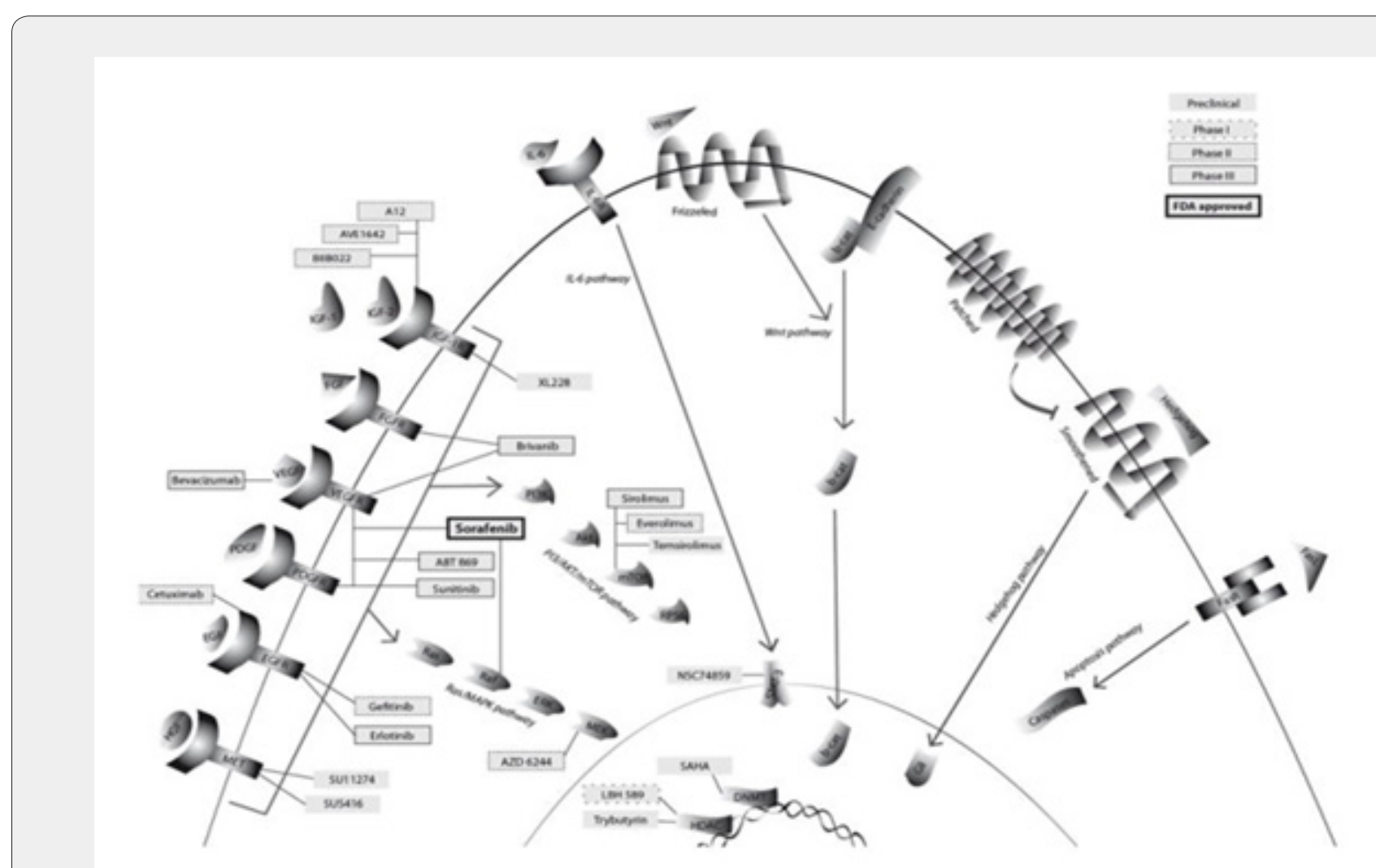

Figure 1: Reproduced from Villanueva A, Newell P, Chiang DY, Friedman SL, Llovet JM. Genomics and signaling pathways in hepatocellular carcinoma. Semin Liver Dis. 2007 Feb;27(1):55-76.

Relevant signaling pathways target of therapies, and molecular targeted therapies currently tested in preclinical and clinical trials in HCC. Only sorafenib has been approved by regulatory agencies. 


\section{Pathways related with cell Survival}

Wnt signaling is a frequently activated pathway observed in HCC as well as in other cancers [2]. Wnt ligands stimulate Frizzled receptors (FZD) with or without coreceptors, so that either the canonical $\beta$-catenin pathway or the noncanonical c-Jun $\mathrm{N}$-terminal kinase (JNK) and protein kinase C (PKC) pathways are activated. In contrast, secreted Frizzled-related proteins can bind to Wnt ligands and block the activation of FZD. If the pathway is activated, $\beta$-catenin can uncouple from E-cadherin and translocates into the nucleus where it regulates oncogenes like c-myc, survivin and cyclin D. $\beta$-catenin gene (CTNNB1) mutations represent the second highest frequency of known mutations in HCC and define a molecular subclass of HCC associated to activation of
Wnt signaling [8]. General activation of the pathway has been described in one third of HCCs, but mutations occur in only $17 \%$ of cases [9]. Interestingly the Wnt signaling cascade is mainly activated in HCV positive HCCs.

The hedgehog $(\mathrm{Hh})$ signaling pathway is another crucial pathway described in HCC among other cancers. It is important for embryonic liver development and regeneration, stem cell biology and cell differentiation. Sonic $\mathrm{Hh}(\mathrm{SHH})$ and smoothened (SMO) are frequently overexpressed in HCC, activate the pathway and contribute to hepatocarcinogenesis. Its negative regulator Hh-interacting protein (HHIP) is downregulated in HCC due to promoter hypermethylation and/or loss of heterozygosity ( $\mathrm{LOH})$ (Figure 2).

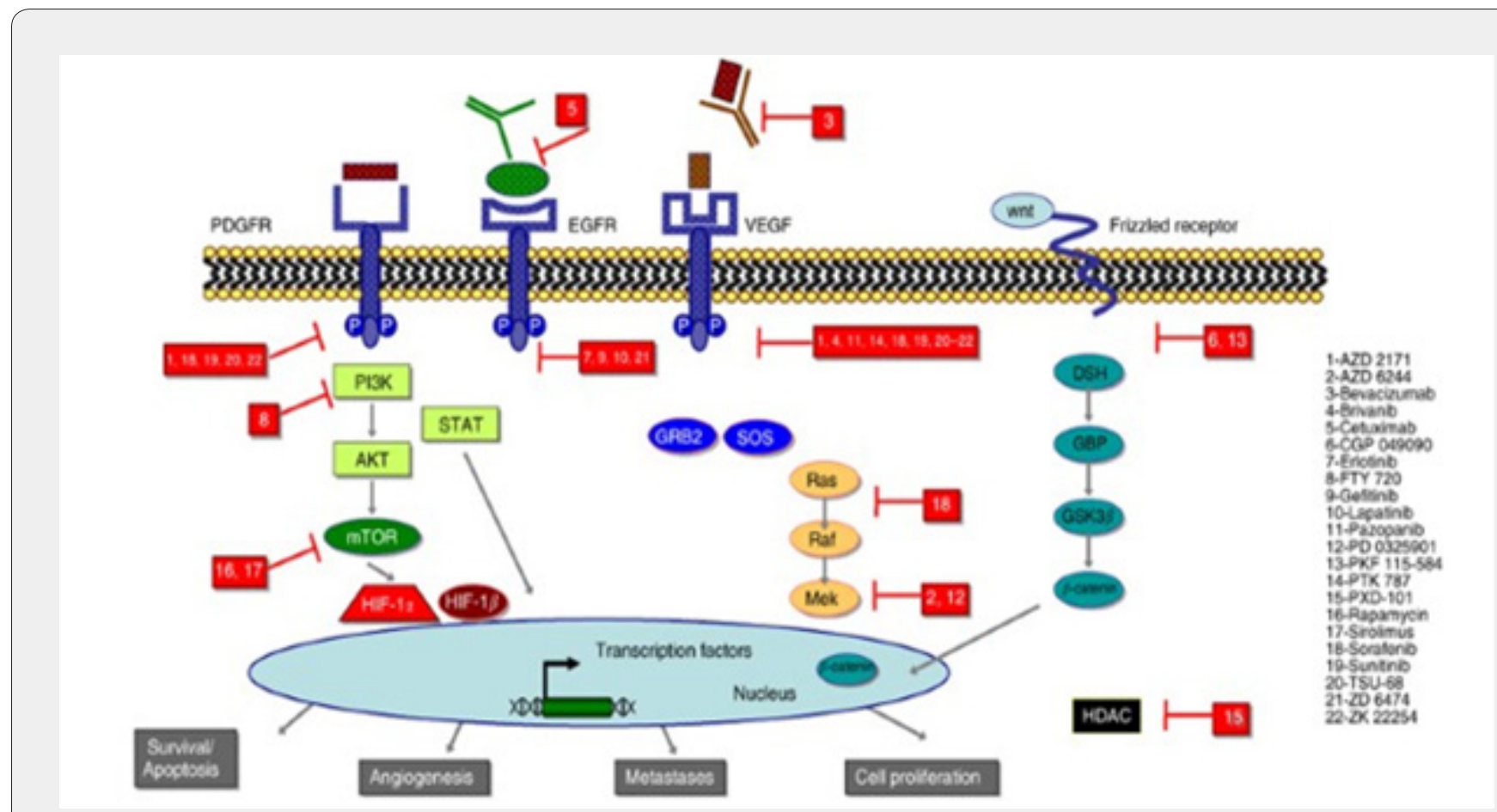

Figure 2: Reproduced from Greten T, Korangy F, Manns $\mathrm{M}$ et al. Molecular therapy for the treatment of hepatocellular carcinoma. Br J Cancer 100, 19-23 (2009).

\section{Pathways related to cell Proliferation}

Hepatocyte growth factor (HGF) and its tyrosine protein kinase receptor c-Met (mesenchymal-epithelial transition factor) pathway play an important role in cellular responses, such as invasive growth in development and cancer, tissue regeneration, angiogenesis, proliferation, and migration. HGF overexpression and aberrant c-Met activation has been reported in HCC due to c-Met amplification, mutation, and transcriptional upregulation of HGF dependent autocrine loops [2]. C-Met expression showed an inverse correlation to patient survival and c-Met silencing led to decreased cell migration and proliferation in an in vitro HCC model. HGF expression was higher in cirrhotic livers and high serum levels were associated with worse outcome after tumor resection.

Activation of the insulin-like growth factor (IGF) signaling pathway is another common event in HCC. Overexpression of IGF1 and IGF-2 receptor (IGF-1R/2R) and silencing of the IGF binding proteins (IGFBP-1-5) contribute to proliferation, anti-apoptosis, and invasive behavior. The binding of the ligands IGF-1 and IGF-2 to IGF-1R results in the activation of the intrinsic tyrosine kinase domain, initiating various signaling pathways, including the PI3K/ Akt pathway and Ras/MAPK pathway. The PI3K/PTEN/Akt/mTOR 


\section{Cancer Therapy \& Oncology International Journal}

pathway itself is involved in many cellular processes including proliferation, differentiation, apoptosis, cell cycle progression, cell motility, tumorigenesis, tumor growth, and angiogenesis. Akt activation can happen through the EGF or IGF signaling cascade, prior PI3K activation or PTEN silencing. Aberrant mTOR signaling, another important mediator of the PI3K/PTEN/Akt pathway was present in 50\% of HCCs and is associated with insulin-like growth factor pathway activation, epidermal growth factor up-regulation, and PTEN dysregulation [4]. The tumor suppressor gene PTEN can mediate cell growth, migration and invasion in HCC. The pathway seems to also be involved in invasion and metastasis through overexpression of MMP-9 in HCC.

The EGFR-Ras-mitogen-activated protein kinase (MAPK) pathway mediates cellular responses to growth signals and is frequently dysregulated in cancer. It is a complex pathway and regulator of cell growth and division. EGF, HGF, PDGF and VEGF are examples of ligands activating the cascade in general, whereas in HCC activation possibly results from aberrant EGFR and IGF signaling or from hypermethylation and subsequent silencing of Ras-binding TSGs (RASSF1A, NORE1A) [10]. Mutations of key regulators of this pathway are rather rare in HCC and seem to play an inferior role as activators.

Most of the pathways described above are involved in either the regulation of cell proliferation or programmed cell death (apoptosis), a major phenomenon in cell biology. In cancer many apoptotic events are altered, and uncontrolled cell proliferation leads to the formation of neoplasms [11]. Apoptosis can be activated either through an intrinsic (e.g. DNA damage) or extrinsic pathway (binding of pro-apoptotic factors to cell surface) leading to the destruction of cells by proteins called caspases. In HCC some proapoptotic molecules are downregulated (Fas, p53, PTEN, Bax, Bid), but the imbalance between cell survival and death is mainly due to overexpression of antiapoptotic signals (Snail, $\beta$-catenin, NF-кB, c-IAP1, Akt, Ras/ERKs, HGF, and EGFR ligands) [12].

\section{Genetic Alterations}

Chromosomal amplifications and deletions, mutations and genomic instability are the most important structural changes in HCC. Mutations in p53 and $\beta$-catenin are probably best described and most prevalent in HCC. The tumor suppressor gene p53 is mutated in $27 \%$, $\beta$-catenin in $0-44 \%$ of cases, leading to a disruption of cell cycle control, DNA repair, apoptosis and proliferation, and the activation of the Wnt pathway. Mutations in AXIN1, K-Ras, and PI3KCA have been described, but are less frequent [4]. Results of whole genome deep sequencing are expected to change the portrait of critical mutations in HCC. Chromosomal amplifications (1q, 6p, 8q, 17q and 20q) and deletions (4q, 8p, 13q, 16q, and 17p) alter several candidate genes that have been identified to be crucial for hepatocarcinogenesis: c-myc, PTEN, E-cadherin, AXIN1, p53, Rb1, FGF3/4, Cyclin D1, and IGF-2R [13]. Among others the activation of telomerase in
HCC is associated with increased genomic instability. Its activity is increased in more than $80 \%$ of HCCs resulting in telomere length alteration [14].

\section{Epigenetic Alterations}

Epigenetic alterations are heritable changes in the pattern of gene expression mediated by mechanisms other than alterations in the DNA sequence. They consist of DNA methylation, histone modifications and nucleosome remodeling. Genome wide hypomethylation is a common event in cancer leading to genomic instability, which has also been described for HCC. Hypermethylation of $\mathrm{CpG}$ islands within the regulatory regions of genes usually leads to gene silencing. Hypermethylation and silencing of important tumor suppressor genes like p16, RASSF1, IGFBP3, E-cadherin and SOCS 1 have been reported among many others in HCC. Conversely, hypomethylation of promoter regions and reactivation of oncogenes (c-myc) was demonstrated in HCC [2]. Histone modifications are more complex alterations on the N-terminal tails (methylation, acetylation, ubiquination, etc.) that can regulate gene expression either directly or through chromatin remodeling. Only very few genes regulated by histone modifications have been described in HCC $[15,16]$. In addition to this, HBx antigen has been shown to recruit the enzyme responsible for histone deacetylation (HDAC1) and to transcriptionally repress IGFBP-3.

\section{Small RNAs}

MicroRNAs (miRNAs) are small RNA molecules, approximately 22 nucleotides long, that negatively control their target gene expression post transcriptionally. Their role in cancer is of increasing importance, since they can act as oncogenes or tumor suppressor genes. In HCC several miRNAs have been shown to be dysregulated. Some function as oncogenes and inhibit apoptosis (miR-221), promote cell invasion (miR-9) or silence c-Met and uPA-expression and by that inhibit migration and proliferation (miR23b). Others seem to have a tumor suppressor gene like function (miR-101, 195, 122, 338) and are silenced in HCC. Downregulation of miR-122 was reported to correlate with suppression of the hepatic phenotype and gain of metastatic properties. Just recently, the upregulation of Lin28b in HCC and its consequent repression of miR-let7 has been described to be associated with advanced disease and poor clinical outcome [17].

\section{Molecular Therapies}

Anti-cancer drugs targeting cancer specific molecules or gene alterations are designated molecular targeted therapies. Herein the protein kinase inhibitors represent the biggest group in oncology, followed by monoclonal antibodies. Many molecular alterations in HCC display potential targets for molecular targeted drug therapy in HCC. Effective compounds are urgently needed to stop the progress or relapse of this fatal disease, particularly in 


\section{Cancer Therapy \& Oncology International Journal}

advanced and incurable tumors, but also in the adjuvant setting after resection of HCC.

Sorafenib was the first compound that significantly improved the survival of patients with advanced HCC [4]. This multikinase inhibitor is active against Raf-1, VEGFR2, B-Raf, PDGFR and c-Kit as well as other receptor tyrosine and serine threonine kinases. Preclinical models showed significant anti-tumoral activity of sorafenib by reducing cell viability and inducing apoptosis in vitro and in vivo. Later, a phase II trial was conducted that demonstrated clinical benefit in treated patients.

This led to the design of a large phase III clinical trial (SHARP, $n$ $=602$ ) that was terminated after the first interim analysis showed significant survival benefit for patients treated with sorafenib compared to placebo (10.7 versus 7.9 months, respectively) [4]. The FDA (US Food and Drug Administration) and EMEA (European Medicine Agency) approval of sorafenib followed shortly after, and a consensus of several HCC expert panels recommended that sorafenib should be the standard of care for the treatment of advanced HCC, as well as the control-arm that novel compounds for the treatment of HCC need to be tested against in future clinical trials [18]. These results truly are a milestone in the treatment of HCC and stimulated the search for similar compounds targeting other molecular alterations, in particular because combination therapies are postulated to be a promising approach through synergistic anti-tumoral effects [18].

Erlotinib, Gefitinib, Laptinib and Vandetanib are tyrosine kinase inhibitors targeting EGFR that have all been tested in clinical trials for HCC [19]. Erlotinib showed anti-tumoral activity in experimental models and clinical studies of HCC. Also, treatment in combination with the VEGF-Inhibitor Bevacizumab showed promising results and is currently under investigation in a phase II trial compared to sorafenib alone. Although Gefitinib showed activity against HCC in preclinical models, the published data of the clinical trial reported no activity in patients [13]. Lapitinib and Vandetanib are two dual blocking molecules against Her2/neu and VEGFR, respectively. Vandetanib inhibited HCC cell proliferation, migration and invasion in vitro, and is currently under investigation in a phase II trial for inoperable HCC. Lapatinib has been tested in clinical trials but showed only minimal antitumoral activity, possibly because Her2/neu overexpression is rather uncommon in HCC. Cetuximab, a monoclonal antibody inhibitor of EGFR, inhibited cell proliferation and induced cell cycle arrest and apoptosis in an HCC cell line.

MTOR-inhibitors are of increasing interest for the treatment of HCC, particularly since activation of the PI3K/Akt/mTOR pathway was reported for as many as $50 \%$ of HCCs [20]. The same study successfully inhibited tumor growth and improved survival with the mTOR-inhibitor everolimus in vitro and in a xenograft model. Ongoing clinical trials are studying everolimus alone and in combination to sorafenib for HCC. The VEGF inhibitor bevacizumab is currently under investigation in a combination with the mTOR-inhibitor rapamycin (see http:// www.clinicaltrials.gov), an analog that has activity against HCC in xenograft models [21]. Temsirolimus is a novel mTOR-inhibitor that was only tested in a preclinical model for HCC.

Angiogenesis and the overexpression of angiogenic factors such as VEGF, PDGF, FGF-2, or angiopoietin are early and frequent events in hepatocarcinogenesis [22]. Therefore, blocking angiogenesis directly or indirectly by targeting microvascular endothelial cells or protein activators of angiogenesis, respectively, is an important strategy in preventing tumor progression. The humanized monoclonal antibody bevacizumab (anti-VEGF) is approved for the treatment of breast cancer and colorectal liver metastases and showed modest anti-tumoral activity in a phase I/II trial of 33 selected patients and in a study that combined treatment with the chemotherapy's gemcitabine and oxaliplatin [23].

Vatalanib, a small molecule inhibitor of VEGFR1 and -2 has only been tested in vitro up to now, where it inhibited cell proliferation and induced apoptosis. The multikinase inhibitor sunitinib targets the angiogenic factors VEGFR1,-2 and PDGFR and is approved for renal cell carcinoma and gastrointestinal stromal tumors. Some anti-tumoral activity against HCC has been reported in two phase II studies, but an important reported sideeffect is gastrointestinal bleeding, and potentially life-threatening complications [24]. Brivanib is a small molecule inhibiting FGF and VEGFR that is currently under investigation in phase III trials after showing promising results as a second line agent after sorafenib failure. TSU-68 is a small molecule inhibitor of the angiogenic receptor tyrosine kinases Flk-1/KDR, PDGFR $\beta$, and FGFR1, currently in phase I/II clinical trials for HCC, although there has been no in vitro data published so far.

The IGF signaling axis is frequently activated in HCC and several IGF-1R tyrosine kinase inhibitors and molecular antibodies have been tested preclinically for HCC and other cancers. BIIB022 and AVE 1642 are two novel drugs in phase I/ II studies as monotherapies or in combination with sorafenib and erlotinib, respectively, whereas the monoclonal antibody IMC-A12 has been investigated only in combination with sorafenib in a phase II clinical trial. The hedgehog and Wnt- $\beta$-catenin pathway are commonly deregulated in hepatocarcinogenesis, but effective drugs are still under development. The hedgehog antagonist cyclopamine effectively inhibited hepatoblastoma cell growth and induced apoptosis. RNA interference-mediated knockdown of CDH17 inactivated Wnt signaling by inhibiting proliferation of primary and highly metastatic HCC cell lines in vitro and in vivo. Both preclinical studies demonstrate very nicely how important it is to find clinically usable drugs for the inhibition of the Wntsignaling and hedgehog pathway. So far there are no ongoing clinical trials with these compounds in HCC.

One further approach in the treatment of HCC is targeting the epigenetic changes that inactivate important tumor suppressor 
genes or pathway antagonists. These epigenetic drugs consist of two major groups: demethylating agents and Histone Deacetylase Inhibitors (HDACi). Both are able to reexpress epigenetically silenced genes, either by demethylation of methylated promoter regions or by histone acetylation [25]. Several novel compounds (MS-275, SAHA, Tributyrin, Valproic Acid) have been tested preclinically in HCC and other cancers showing promising results mainly when used as combination therapy. The novel HDACi LBH589 is now tested in combination with sorafenib in a phase I trial for advanced HCC, although preclinical results have only been reported for other cancers. Demethylating agents like Dacogen and Vidaza have first been FDA approved for the treatment of myelodysplastic syndrome and are currently under preclinical and clinical investigation for several other hematological and solid malignancies. Although SAHA and Dacogen have been tested successfully in a preclinical setting for HCC and other solid tumors, none of these compounds have moved to clinical trials yet.

The potential of antagomirs or reconstitutive miRNA compounds in the treatment of cancer is also a very appealing new approach, with promising preclinical results. Nevertheless, this area needs further exploration before it can be studied in clinical trials. As of November 2020, approved treatments for advanced HCC include the tyrosine kinase inhibitors sorafenib, lenvatinib, cabozantinib, and regorafenib; the VEGFR2 inhibitor ramucirumab; the immune checkpoint inhibitors nivolumab (with or without ipilimumab) and pembrolizumab; and the recently approved PD-L1 inhibitor/VEGF inhibitor combination of atezolizumab plus bevacizumab. Atezolizumab plus bevacizumab is now the default first-line systemic treatment for most patients with advanced HCC and Child-Pugh A liver function, replacing sorafenib and lenvatinib as the preferred standard of care in this setting.

\section{Conclusion}

Molecular targeted therapies for the treatment of cancer including HCC are a very promising approach, clearly demonstrated by the breakthrough results of the multikinase inhibitor sorafenib [26]. The success of sorafenib is proof of the principle that molecular therapy plays an important role in the treatment of advanced HCC. Research focused on understanding the molecular pathogenesis and pathways in HCC over the past years has helped to identify many novel potential targets that need to be further evaluated in preclinical and clinical trials. Nonetheless, the ultimate biomarker to identify treatment responses has not been identified. Increasing evidence now justifies the testing of combination therapies targeting different molecules also in adjuvant settings. Even the combination of novel epigenetic drugs or anti-miRNA compounds with small molecules like sorafenib could be a promising new approach in treating this complex disease. Last but not least, the increasing knowledge of molecular subclasses of HCC will help to identify which patients will benefit best from certain compounds, an important step towards personalized medicine in the treatment for HCC. The identification of robust molecular subclasses and specific oncogenic loops is of special relevance in HCC since high molecular heterogeneity is observed. Certain molecular targeted therapies could be evaluated in patients selected according to their molecular profile in order to optimize clinical benefits for specific molecular subgroups.

\section{How I treat Hepatocellular Carcinoma at the Cochin Cancer Research Centre}

Most patients with hepatocellular carcinoma (HCC) also have liver cirrhosis and thus have dual competing causes of mortality. Whenever I meet with a patient with HCC, I first assess the severity of the underlying cirrhosis before providing a treatment recommendation. A challenge in managing patients with advanced HCC is that most of the clinical trials supporting the use of approved systemic therapies were conducted in populations of patients with good liver function. In this commentary, I explore how I currently make systemic therapy decisions for patients with advanced HCC and more severe cirrhosis.

\section{Child-Pugh Scoring and Clinical Trials of Systemic Therapy in Advanced HCC}

The Child-Pugh scoring system uses 5 parameters-ascites, bilirubin, albumin, prothrombin time, and encephalopathy-to classify severity of liver cirrhosis. Patients with the lowest scores (and, as such, the least severe liver disease) are classified as having Child-Pugh A disease; those with more severe cirrhosis are classified as having Child-Pugh B or C liver function. As may be expected, patients with Child-Pugh B liver function have significantly decreased OS compared with patients with ChildPugh A liver function. Because of this, patients with Child-Pugh B liver function are often excluded from clinical trials of novel therapies for HCC due to their poor prognosis. As a result, most approved systemic therapies for HCC have only been prospectively studied in Child-Pugh A populations, with little information to guide treatment in patients with more severe levels of liver impairment.

A challenge in managing patients with advanced HCC is that most of the clinical trials supporting the use of approved systemic therapies were conducted in populations of patients with good liver function. In this commentary, I explore how I currently make systemic therapy decisions for patients with advanced HCC and more severe cirrhosis. Before I make a treatment recommendation for a patient with advanced HCC and Child-Pugh B liver function, I try to assess liver reserve beyond Child-Pugh score. There is a subset of patients with Child-Pugh B liver function that is driven by cancer-related hyperbilirubinemia and hypoalbuminemia rather than decompensated cirrhosis; these patients generally had near-normal liver function and platelets prior to their HCC. 
Such patients can often tolerate systemic therapy better than patients with Child-Pugh B liver function driven by cirrhosis, and I treat them similarly to patients with Child-Pugh A liver function. In some cases, their liver function may actually improve if their tumor responds to systemic therapy.

For the majority of patients with Child-Pugh B liver function, however, I am uncomfortable using atezolizumab plus bevacizumab in the frontline setting. Although this combination was shown in the phase III IMbrave150 study to significantly prolong OS vs sorafenib in patients with Child-Pugh A liver function, the safety of this combination has not been established in patients with Child-Pugh B disease. A major concern with this combination in the Child-Pugh B population is hemorrhagic complications related to bevacizumab. In historical phase II trials of bevacizumab that included patients with HCC and Child-Pugh B liver function, hemorrhagic complications were observed in up to $19 \%$ of patients.

I am comfortable using sorafenib in patients with Child-Pugh $\mathrm{B}$ cirrhosis and consider this agent to be a reasonable frontline treatment option for these patients. This agent has been studied both retrospectively and prospectively in patients with Child-Pugh B disease, and has generally been found to be safe, tolerable, and effective in this population. Although the sorafenib label does not provide a dose recommendation for these patients, I dose reduce this agent to $200 \mathrm{mg}$ twice daily in this population.

I am slightly less comfortable using lenvatinib in the frontline setting for patients with Child-Pugh B cirrhosis because safety is not as well established in this population. Furthermore, in the phase III REFLECT study comparing lenvatinib with sorafenib in patients with Child-Pugh A liver function, patients receiving lenvatinib had higher rates of liver function-related adverse events, including hepatic encephalopathy.

There are limited retrospective data with cabozantinib to support use of this agent in patients Child-Pugh B liver function. What data there are come from the phase III CELESTIAL trial, for which patients were required to have Child-Pugh A liver function to enroll. Data in the Child-Pugh B setting therefore come from those patients whose liver function deteriorated during the trial; this population may not be reflective of most patients with ChildPugh B cirrhosis. Per the package insert, cabozantinib should be dosed at $40 \mathrm{mg}$ in patients with Child-Pugh B disease.

Anti-PD-1 immunotherapy with nivolumab has also been studied in patients with HCC and Child-Pugh class B7 or B8 liver function without ascites or encephalopathy in a prospective cohort of the CheckMate-040 study, as well as in a broader Child-Pugh B retrospective cohort study from the University of California at San Francisco. These studies indicate that nivolumab has encouraging efficacy and tolerability in patients with HCC and Child-Pugh B liver function. As nivolumab is generally better tolerated than sorafenib, based on the limited data available I think nivolumab monotherapy is a compelling choice in the frontline setting for patients with HCC and Child-Pugh B liver function. However, Nivolumab is yet to find a place in the hospital formulary of the Cochin Cancer Research Centre.

Given the poor prognosis of patients with HCC and ChildPugh C liver function and the high risk of treatment-related liver toxicity, I generally recommend supportive care as the best option for these patients.

\section{Future Perspectives}

The increase in deaths due to hepatocellular carcinoma is a growing concern. The hope is that universal HBV vaccination, the increasing cure rates of HCV infection, and improvements in surveillance will reduce this burden. Hepatocellular carcinoma is a complex disease, frequently associated with cirrhosis; a multidisciplinary approach in specialized clinics is required to maximally influence the course of the disease. The clinical management of hepatocellular carcinoma has improved in the past 10 years, particularly for patients at advanced stages. Other areas of management remain without effective interventions, such as chemoprevention in patients with cirrhosis and adjuvant therapies after surgical resection or ablation. As the number of systemic agents found to be effective in phase 3 trials continues to grow, the challenge is to determine the order of sequential systemic therapy that maximizes the clinical benefit with minimal toxic effects and cost. The prospect of combination therapies and the use of systemic drugs at earlier stages will shape research initiatives for hepatocellular carcinoma in the near future. There must be space for inclusion of these new drugs in the Cancer care formulary of Kerala Cancer Grid without which this seemingly unsurmountable problem will linger as a Damocles sword hanging over our heads.

The current scenario of systemic therapy in HCC is likely to change in the near future. If the encouraging data on immunotherapy materializes, a major challenge would be to evaluate the potential benefit of sequenced strategies or combination between active agents. Undoubtedly, proper patient selection and an accurate and critical interpretation of trials results will remain crucial.

\section{References}

1. EASL-EORTC (2018) Clinical Practice Guideline Management of hepatocellular carcinoma. J Hepatol 56: 908-943.

2. Villanueva A, Newell P, Chiang DY, Friedman SL, Llovet JM (2007) Genomics and signaling pathways in hepatocellular carcinoma. Semin Liver Dis 27:55-76.

3. Farazi PA, DePinho RA (2006) Hepatocellular carcinoma pathogenesis: from genes to environment. Nat Rev Cancer. 6(9): 674-687. 


\section{Cancer Therapy \& Oncology International Journal}

4. Llovet JM, Burroughs A, Bruix J (2003) Hepatocellular carcinoma. Lancet 362: 1907-1917.

5. Feitelson MA, Lee J (2007) Hepatitis B virus integration, fragile sites, and hepatocarcinogenesis. Cancer Lett. 252(2):157-170.

6. El-Serag HB, Rudolph KL (2007) Hepatocellular carcinoma: epidemiology and molecular carcinogenesis. Gastroenterology. 132(7): 2557-2576.

7. Vogelstein B, Kinzler KW (2004) Cancer genes and the pathways they control. Nat Med 10(8):789-799.

8. Chiang DY, Villanueva A, Hoshida Y, Judit Peix, Philippa Newell et al. (2008) Focal gains of VEGFA and molecular classification of hepatocellular carcinoma. Cancer Res 68(16): 6779-6788.

9. Laurent-Puig P, Legoix P, Bluteau O, J Belghiti, D Franco, et al. (2001) Genetic alterations associated with hepatocellular carcinomas define distinct pathways of hepatocarcinogenesis. Gastroenterology. 120(7): 1763-1773.

10. Calvisi DF, Ladu S, Gorden A, Miriam Farina, Elizabeth A Conner et al. (2006) Ubiquitous activation of Ras and Jak/Stat pathways in human HCC. Gastroenterology. 130(4): 1117-1128.

11. Hanahan D, Weinberg RA (2000) The hallmarks of cancer. Cell 100(1): 57-70.

12. Fabregat I, Roncero C, Fernandez M (2007) Survival and apoptosis: a dysregulated balance in liver cancer. Liver Int 27(2): 155-162.

13. Minguez B, Tovar V, Chiang D, et al. (2009) Pathogenesis of hepatocellular carcinoma and molecular therapies. Curr Opin Gastroenterol 25(3): 186-194.

14. Farazi PA, Glickman J, Jiang S, et al. (2003) Differential impact of telomere dysfunction on initiation and progression of hepatocellular carcinoma. Cancer Res 63: 5021-5027.

15. Zhang C, Li H, Zhou G, et al. (2007) Transcriptional silencing of the TMS1/ASC tumour suppressor gene by an epigenetic mechanism in hepatocellular carcinoma cells. J Pathol 212(2): 134-142.
16. Kondo Y, Shen L, Suzuki S, et al. (2007) Alterations of DNA methylation and histone modifications contribute to gene silencing in hepatocellular carcinomas. Hepatol Res 37(11): 974-983.

17. Viswanathan SR, Powers JT, Einhorn W, et al. (2009) Lin28 promotes transformation and is associated with advanced human malignancies. Nat Genet. 41(7): 843-848.

18. Llovet JM, Di Bisceglie AM, Bruix J, et al. (2008) Design and endpoints of clinical trials in hepatocellular carcinoma. J Natl Cancer Inst. 100(10):698-711.

19. Llovet JM, Bruix J (2008) Molecular targeted therapies in hepatocellular carcinoma. Hepatology. 48(4):1312-1327.

20. Villanueva A, Chiang DY, Newell P, et al. (2008) Pivotal role of mTOR signaling in hepatocellular carcinoma. Gastroenterology. 135(6): 1972-1983.

21. Newell P, Toffanin S, Villanueva A, et al. (2009) Ras pathway activation in hepatocellular carcinoma and anti-tumoral effect of combined sorafenib and rapamycin in vivo. J Hepatol. 51(4): 725-733.

22. Ribatti D, Vacca A, Nico B, et al. (2006) Angiogenesis and antiangiogenesis in hepatocellular carcinoma. Cancer Treat Rev. 32(6): 437-444.

23. Zhu AX, Blaszkowsky LS, Ryan DP, et al. (2006) Phase II study of gemcitabine and oxaliplatin in combination with bevacizumab in patients with advanced hepatocellular carcinoma. J Clin Oncol 24(12): 1898-1903.

24. Faivre S, Raymond E, Boucher JY, et al. (2009) Safety and efficacy of sunitinib in patients with advanced hepatocellular carcinoma: an open label, multicenter, phase II study. Lancet Oncol 10 (8): 794-800.

25. Sharma S, Kelly TK, Jones PA (2009) Epigenetics in Cancer Carcinogenesis 27-36.

26. Llovet JM, Ricci S, Mazzaferro V, et al. (2008) Sorafenib in advanced hepatocellular carcinoma. N Engl J Med 359(4): 378-390.

Your next submission with Juniper Publishers will reach you the below assets

- Quality Editorial service

- Swift Peer Review

- Reprints availability

- E-prints Service

- Manuscript Podcast for convenient understanding

- Global attainment for your research

- Manuscript accessibility in different formats

( Pdf, E-pub, Full Text, Audio)

- Unceasing customer service

Track the below URL for one-step submission https://juniperpublishers.com/online-submission.php 